\title{
Circuito simplificado para digitalizar las señales de sensores capacitivos aplicando un algoritmo de baja complejidad
}

Simplified circuit to scan capacitive sensors aplying low complexity algorithm

ÓSCAR ALEXÁNDER BELLÓN-HERNÁNDEZ

Ingeniero Electrónico

Mg. en Ingeniería de Control Industrial

Grupo de Investigación GIMAC

Universidad de Boyacá, Colombia

oabellon@uniboyaca.edu.co

Recibido: 13/12/2013

Aceptado: 13/02/2014 


\title{
RESUMEN
}

En este documento se describe un método para digitalizar las medidas suministradas por sensores capacitivos, adaptándolas para ser procesadas desde dispositivos programables. En comparación con los procedimientos estandarizados para medir sensores capacitivos, el circuito utilizado y el algoritmo a partir del cual se calculó el valor de capacitancia, son más simples y presentaron un buen grado de precisión para aplicaciones en sistemas mecatrónicos con constante de tiempo relativamente grande. Los puentes de impedancia que se encuentran en la literatura clásica sobre instrumentación requieren fuentes de corriente alterna adicionales, con frecuencias que deben adaptarse manualmente, a la vez que deben ser monitorizados parámetros de difícil seguimiento, tales como fase, amplitud, magnitudes de impedancia. Tales métodos requieren de instrumentación especializada para determinar con precisión los valores de capacitancia y en el caso de proyectos mecatrónicos, se aumenta la complejidad de los circuitos electrónicos. El método propuesto simplifica considerablemente el diseño de sistemas mecatrónicos basados en sensores capacitivos.

Palabras clave: Instrumentación, sensores capacitivos, adecuación de señales.

\begin{abstract}
This document describes a method to digitize the measurements supplied by capacitive sensors adapting them to be processed from programmable devices. In comparison with standard procedures for measuring capacitive sensors, circuit used and the algorithm from which we calculated the value of capacitance, they are simpler and showed a good degree of accuracy for applications with relatively large time constant mechatronic systems. Links of impedance found in classical literature on instrumentation, require additional power sources $\mathrm{AC}$, with frequencies that should be adapted manually, while should be monitored parameters difficult to track, such as phase, amplitude and impedance magnitudes. Those methods require specialized instrumentation to accurately determine the values of capacitance and mechatronic projects, the complexity of electronic circuits are increased. The proposed method significantly simplifies the design of mechatronic capacitive sensor-based systems.
\end{abstract}

Keywords: Instrumentation, capacitive sensors, signals adaptation. 


\section{INTRODUCCIÓN}

Los sensores capacitivos son transductores eléctricos cuya capacitancia varía en función de alguna magnitud física relacionada. Se utilizan ampliamente para monitorizar variables tales como posición angular, distancia lineal, humedad relativa, concentración de sustancias en fluidos dieléctricos, nivel de líquido en tanques y reservorios, entre otros (Baxter, 1997; Chatzandroulis, Tsoukalas, y Neukomm, 2000; Chiang y Huang, 2006; Kung, Lee, y Howe, 1988; Romani et. al., 2004). La forma tradicional de obtener datos de un sensor capacitivo es mediante la aplicación de diferentes circuitos eléctricos constituidos por reactancias, resistencias y fuentes de corriente alterna (Fernández, García, Alonso, Cano, y Solares, 1998), principalmente los puentes de corriente alterna, cuya configuración general puede observarse en la Figura 1.

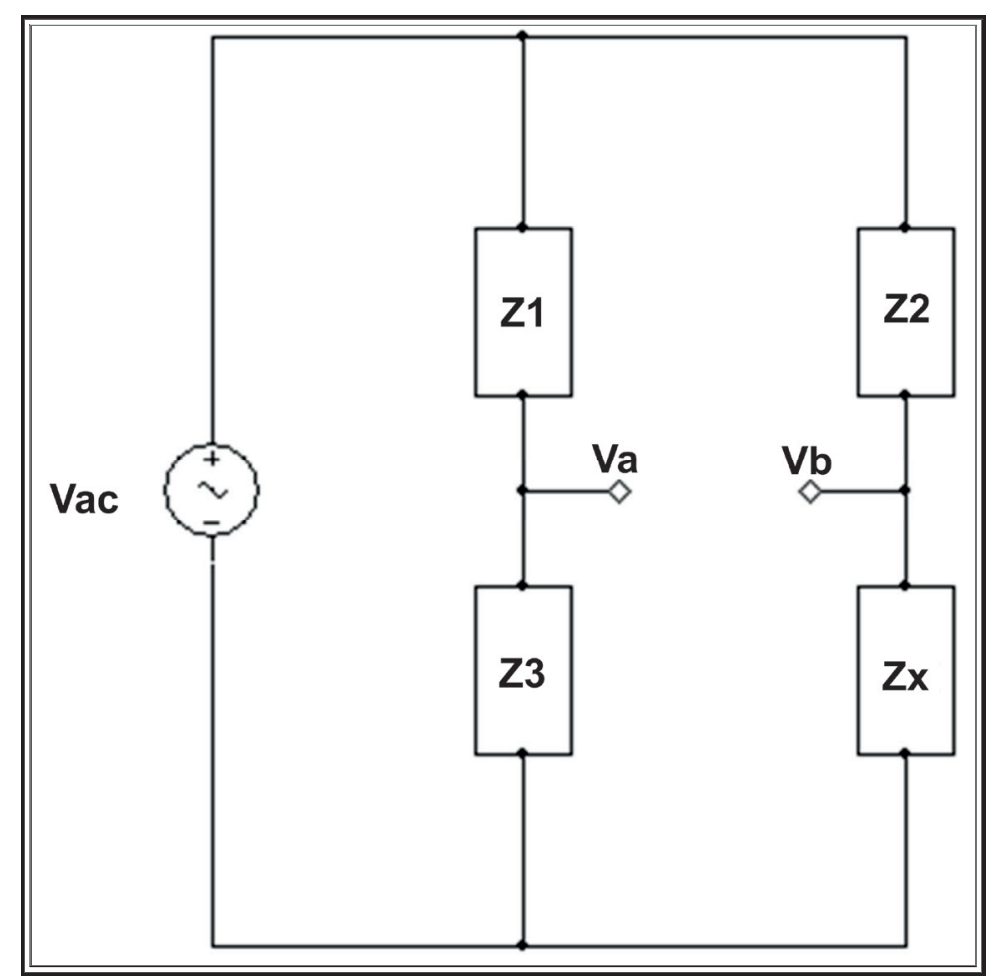

Figura 1. Puente de impedancias utilizado para determinar el valor de una reactancia desconocida 
En esta distribución de impedancias son conocidos los elementos Z1, Z2 y Z3, mientras que la impedancia Zx es la magnitud desconocida. Con instrumentos de medida apropiados (Osciloscopio, generador de funciones) se obtiene la amplitud en voltios y la fase de la tensión sinusoidal a partir del análisis fasorial:

$$
\vec{V} a b=\vec{V} a-\vec{V} b
$$

Algunas de las impedancias conocidas, permiten ser ajustadas manualmente para lograr que la tensión $\vec{V} a b$ sea nula, con lo que la impedancia a determinar puede expresarse fasorialmente como:

$$
\overrightarrow{Z x}=\frac{|Z 1||Z 3|}{|Z 2|} \angle(\theta 1+\theta 3-\theta 2)
$$

La ecuación 2 implica que deben ser ajustadas las amplitudes y fases relativas de las impedancias para lograr la nulidad de la tensión $\vec{V} a b$. Para tal efecto, debe ser adecuado el rango de ajuste de las impedancias y manipularse cuidadosamente la frecuencia de la fuente alterna. El procedimiento se aplica manualmente y requiere de la disponibilidad de dispositivos de medida adecuados y generadores de función confiables. Se fabrican equipos especializados en la medición de reactancias inductivas y capacitivas, disponibles comercialmente, que se basan en la automatización del procedimiento o en la aplicación de técnicas de procesamiento digital de señales. En cualquier caso, dichos equipos son de un costo prohibitivo para lo que un estudiante o diseñador requiere.

Otros métodos también son utilizados para medir capacitancias, tales como los circuitos resonantes, osciladores y los que se basan en carga y descarga de un condensador (Huang, Stott, Green, y Beck, 1988). En los circuitos resonantes, también conocidos como circuitos tanque, se conectan en paralelo un condensador variable y una bobina de inductancia conocida. La resonancia se presenta cuando las reactancias de ambos elementos pasivos son iguales en magnitud. Para obtener una lectura apropiada de un sensor capacitivo en esta configuración es necesario hacer barridos de frecuencia para encontrar la frecuencia de resonancia y en consecuencia, el valor de la capacitancia. Las aplicaciones con sensores capacitivos que usan esta técnica a menudo recurren a circuitos de radiofrecuencia para obtener lecturas (Jaworek y Krupa, 2004).

La medición de capacitancia mediante la monitorización de los tiempos de carga y descarga, es una técnica menos utilizada que las anteriores por considerarse menos precisa. Sin embargo, en procesos físicos donde los tiempos son relativamente largos, comparados con el periodo de las señales que conmutan entre carga y descarga, resultan una alternativa confiable y suficientemente precisa para las necesidades particulares, como por ejemplo en el uso de sensores capacitivos para detección de nivel 
en tanques (Philipp, 1998). Actualmente, con la existencia de dispositivos electrónicos para regular las acciones de conmutación que se necesitan para medir la carga y la descarga, las imprecisiones antes anotadas, se reducen apreciablemente.

Este documento propone un método sencillo para obtener el valor de capacitancia de un sensor capacitivo, basado en un circuito RC serie y en un algoritmo que se construye para medir la constante de tiempo $\tau$, del sistema. Se utilizó una tarjeta programable Arduino Uno, como dispositivo digital para ejecutar el código que aplicaba el algoritmo.

\section{METODOLOGÍA}

La aplicación del método tiene las siguientes partes: montaje del circuito, conexión con la tarjeta programable y la redacción de un código de programación a partir del algoritmo. La primera fase se sustentó en el análisis de la carga y descarga del condensador, cuyo circuito base consistió en una resistencia y un condensador en serie (ver Figura 2), denominado RC serie. Las características de operación del circuito lo hicieron apropiado para trabajar con señales provenientes de la salida digital de la tarjeta Arduino Uno, así como para tomar las lecturas del elemento capacitivo.

\section{Análisis del circuito RC serie}

En primer lugar, se hizo el montaje de un circuito serie compuesto por un condensador y una resistencia (ver Figura 2). Se tiene un sistema de primer orden en el que la función de excitación es una señal rectangular, aplicada a la entrada del circuito (Vi), cuyo periodo es lo suficientemente amplio para que la tensión en las terminales del condensador (Vo) pueda llegar al valor de carga máximo (Vcc). El análisis de la carga y descarga de un condensador, es un fenómeno comúnmente tratado en física, electricidad y electrónica (San Miguel, 2011).

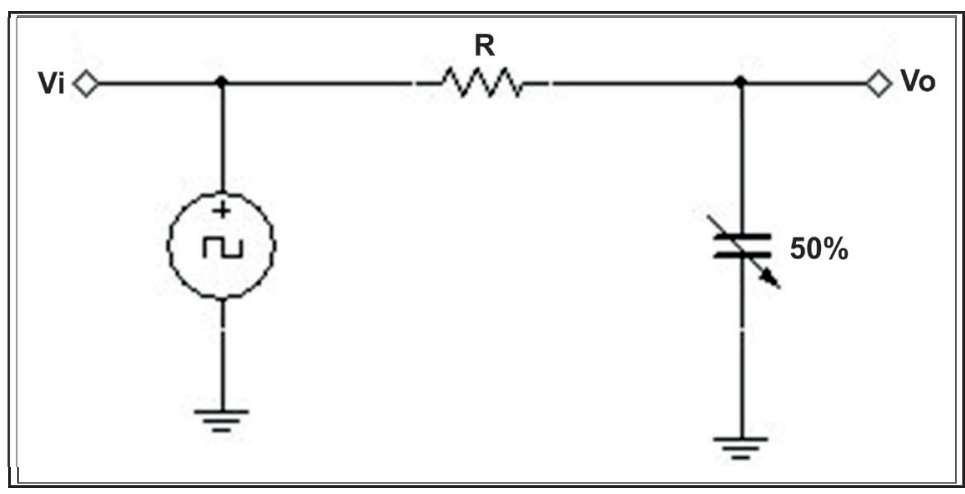

Figura 2. Circuito $R C$ serie con fuente de alimentación tipo onda rectangular 
La constante de tiempo del circuito RC serie es el tiempo que tarda el condensador en alcanzar el 63.2\% aproximadamente del voltaje máximo ante una entrada paso y en función de dicha constante de tiempo se establece el periodo de la onda rectangular (ver Figura 3). La constante de tiempo está definida en términos de la resistencia y de la capacitancia así:

$$
\tau=R C
$$

La resistencia $\mathrm{R}$ es un valor conocido y se selecciona buscando que la constante de tiempo esté dentro del rango de detección de la tarjeta programable. El tiempo mínimo que puede detectarse y que resulta en una medición confiable para determinar la capacitancia, es función del periodo de reloj del dispositivo programable $\left(T_{C L K}\right)$.

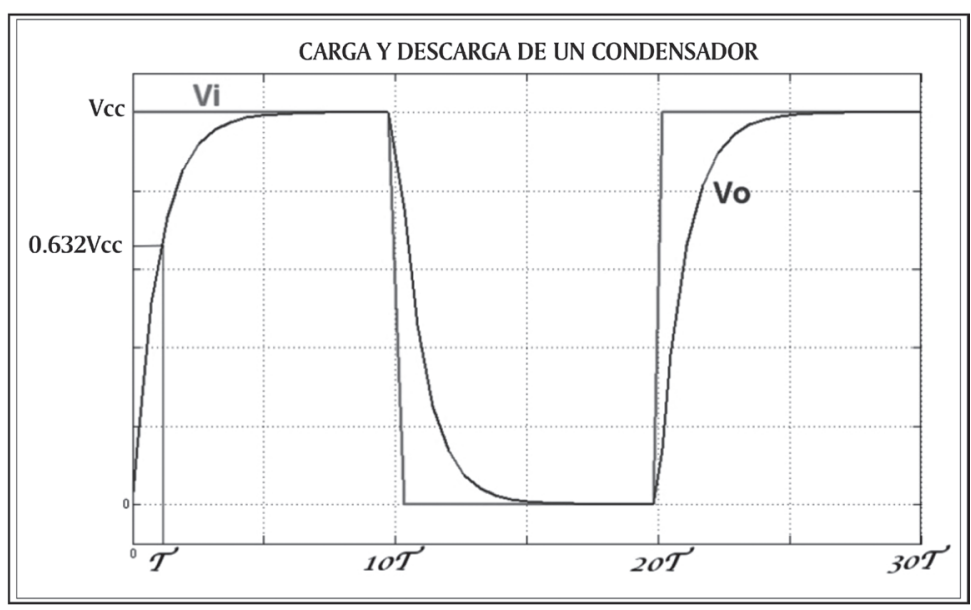

Figura 3. Gráfica comparativa de los voltajes a la entrada (Gris claro) del circuito RC serie y de la tensión en el condensador (Gris oscuro)

Para lograr una mínima precisión en la lectura de la señal del sensor, debe cumplirse la siguiente condición:

$$
T_{C L K} \ll \tau
$$

Para tener una referencia a partir de la cual determinar el grado de precisión de la medida de tiempo, se propuso la siguiente relación: 


$$
T_{C L K} \leq \frac{\tau}{400}
$$

La Figura 3 corresponde a una señal aplicada a la entrada del circuito RC serie, de forma rectangular, con un periodo de al menos 20 veces la constante de tiempo $\tau$. En realidad, no es necesario que la onda de entrada tenga tal periodo, ni que la onda sea simétrica (tiempo en estado alto igual al tiempo en estado bajo). Se puede aplicar una señal rectangular cuya duración en estado alto garantice que la tensión en las terminales del condensador alcance el 63.2\% aproximadamente del voltaje máximo aplicado por la fuente, mientras que la duración en estado bajo permite la descarga completa del condensador, como puede apreciarse en la Figura 4.

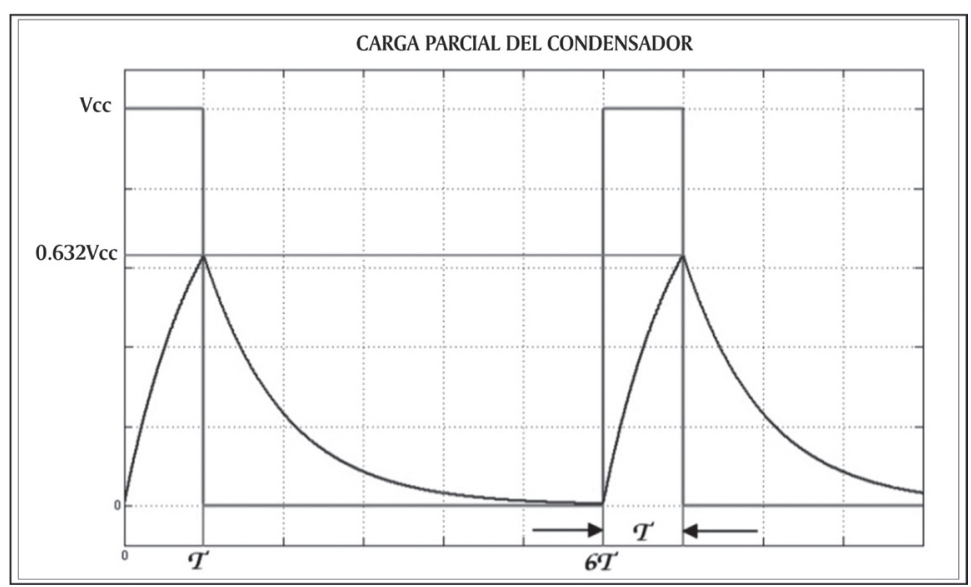

Figura 4. Onda rectangular asimétrica aplicada a la entrada (Gris claro) del circuito RC serie, para cargar el condensador durante un tiempo equivalente a la constante (Gris oscuro)

Una señal rectangular con un periodo de y un ciclo de trabajo de aproximadamente $16.7 \%$, permite la detección de la constante de tiempo.

\section{Conexión a la tarjeta programable Arduino Uno}

Los dispositivos programables, en particular la tarjeta Arduino Uno, trabajan con niveles de tensión binarios para aplicar salidas o entradas digitales. El estado alto corresponde a 5 voltios, aunque es admisible una tensión mínima de 3.3 voltios. El estado bajo es aproximadamente 0 voltios. Un pin de la tarjeta Arduino, programado como salida digital, aplica 5 voltios en estado alto y 0 en estado bajo. Se dispone también de 6 entradas que admiten señales análogas que varían en el rango continuo de 0 a 5 voltios. Estas entradas son conexiones físicas a conversores análogo-digitales de 10 bits de resolución. 
Lo anterior significa que en el rango de voltajes de 0 a 5 voltios, se puede hacer la conversión de la señal a un valor entero mapeado entre 0 y 1023. En la práctica, significa que cada incremento en el voltaje de entrada de $4.88 \mathrm{mV}$, produce el incremento en una unidad en los valores binarios de registro en el conversor A/D. Los voltajes análogos se procesan internamente como valores enteros de 0 a 1023 y en este orden de ideas, se tiene que hacer la conversión para que corresponda al voltaje análogo aplicado.

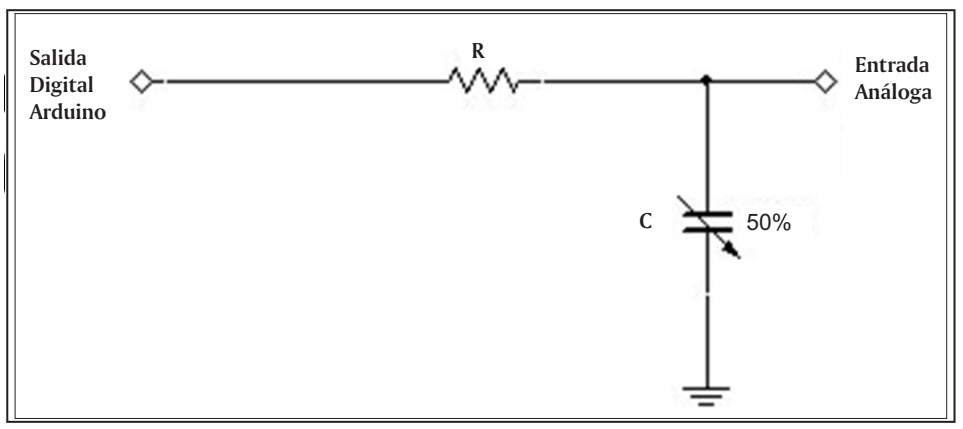

Figura 5. Configuración básica para conexión del circuito $R C$ serie con la tarjeta programable Arduino Uno

La fuente de entrada de señal rectangular, fue aplicada desde un pin de la tarjeta Arduino, configurado como salida digital, mientras que la tensión en las terminales del condensador, se tomó como entrada análoga a la misma tarjeta.

Algoritmo para determinar la capacitancia del sensor capacitivo.

La determinación de la capacitancia, se basa en la medición de la constante de tiempo $\tau$, del circuito $\mathrm{RC}$ serie. Los pasos del algoritmo, son:

1. Asegurar que la carga inicial del condensador sea 0 voltios. Para ello, se debe poner la salida digital de la tarjeta Arduino en estado bajo, para que se reduzca la carga remanente que haya inicialmente.

2. Esperar que la tensión en las terminales del condensador y medida en la entrada análoga de la tarjeta, sea 0 voltios. Cuando se alcance esta condición, se pone la salida digital en estado alto.

3. Se inicia un temporizador, basado en el periodo de reloj del controlador de la tarjeta.

4. Se entra en estado de espera mientras la entrada análoga registra que la tensión en las terminales del sensor capacitivo alcanza el $63.2 \%$ del valor máximo a la entrada, equivalente al voltaje en estado alto. 
5. Se detiene el temporizador al llegar al $63.2 \%$ del voltaje en estado alto, en las terminales del sensor capacitivo. La cuenta del temporizador corresponde a la constante de tiempo $\tau$, del circuito RC serie. A partir de la ecuación 3 y conocido el valor de la resistencia en serie con el sensor, se puede despejar y hallar el valor de la capacitancia del sensor.

6. Vuelve al paso 1.

El proceso puede visualizarse con claridad en el diagrama de flujo presentado en la Figura 6:

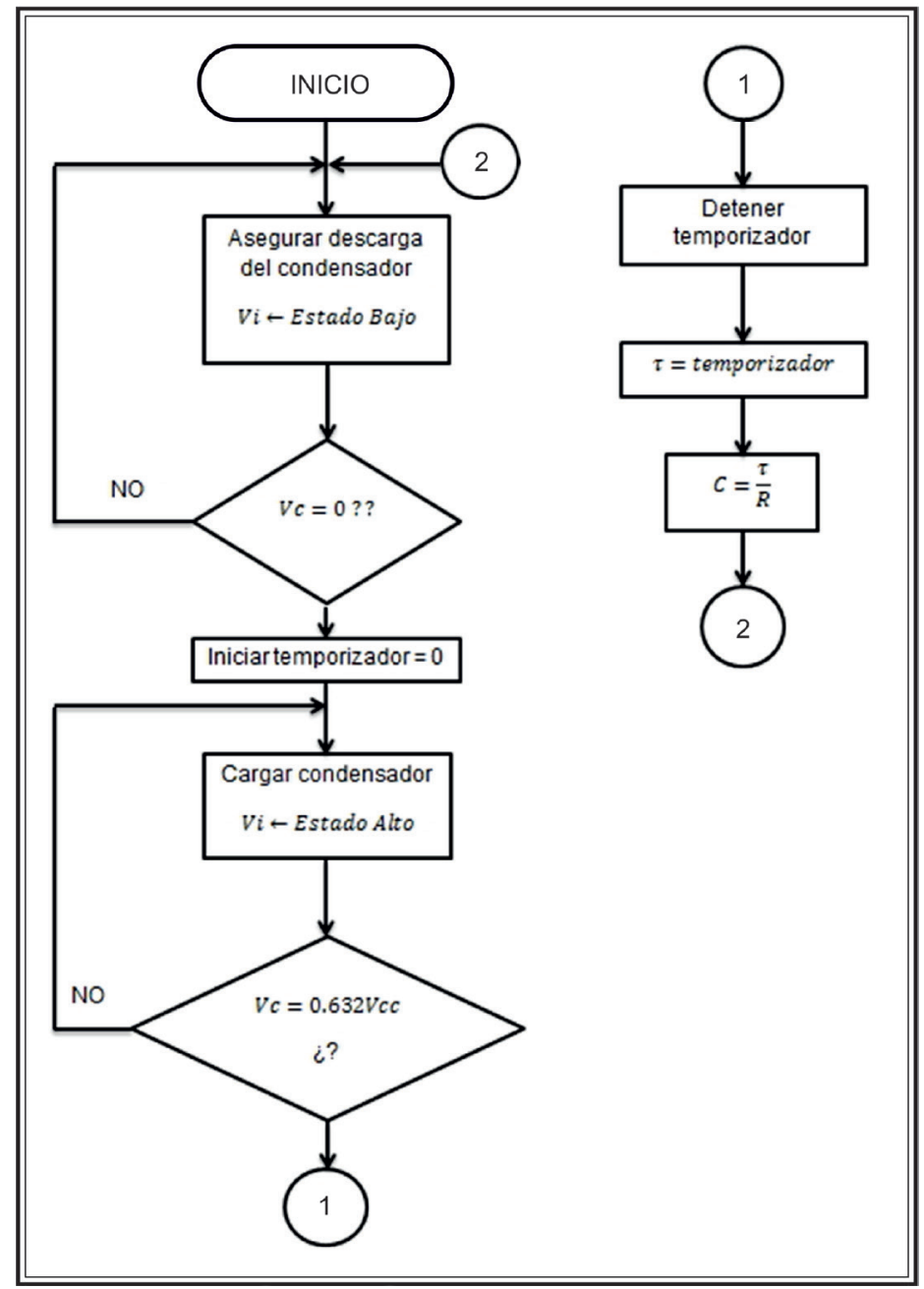

Figura 6. Diagrama de flujo para orientar en el planteamiento del algoritmo 
El código del programa en el entorno Arduino IDE (Entorno Integrado de Desarrollo), puede leerse a continuación:

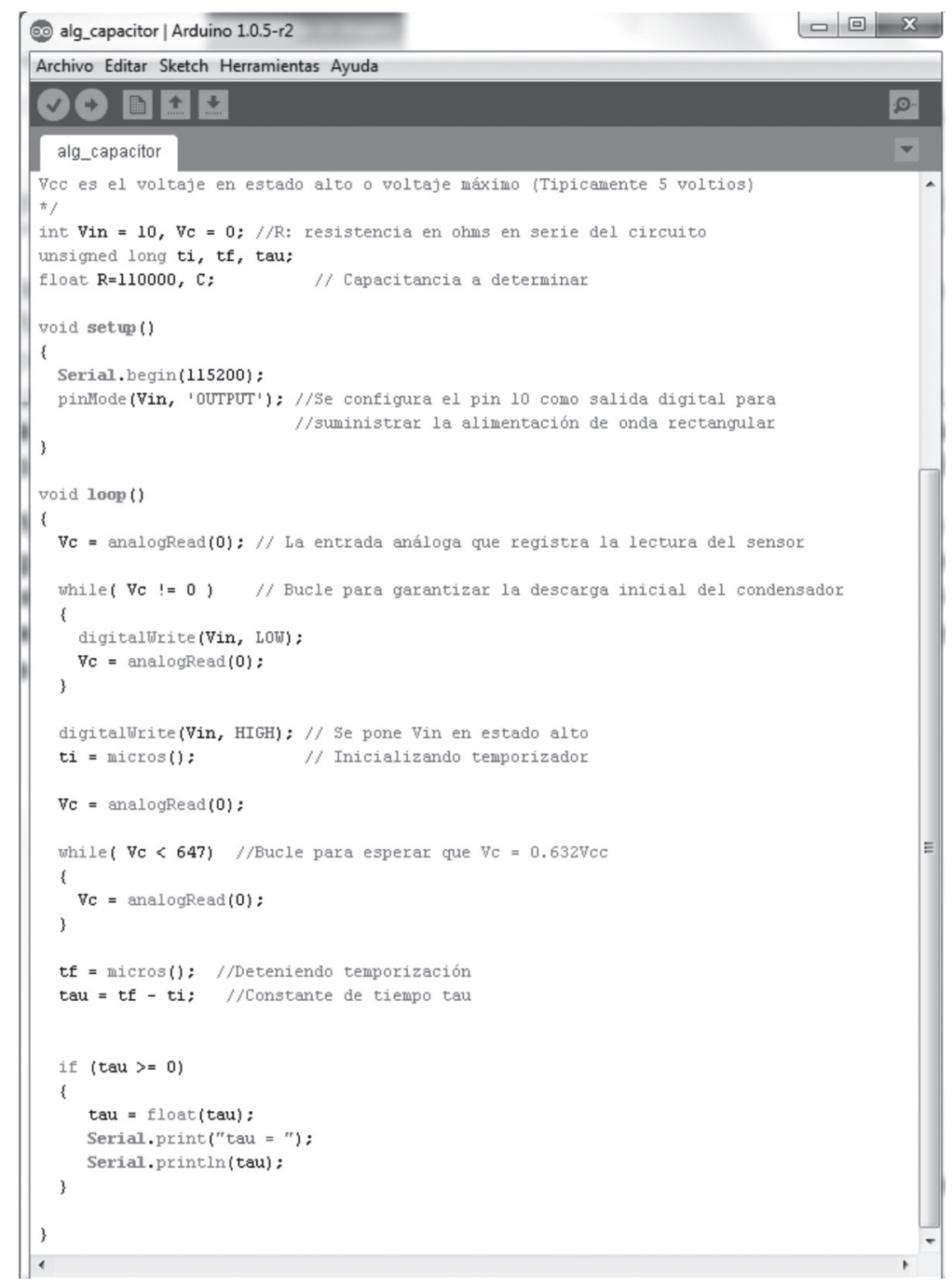

Figura 7. Código del programa en el entorno Arduino IDE que implementa el algoritmo para determinar la capacitancia de un sensor capacitivo 


\section{RESULTADOS Y DISCUSIÓN}

Se utilizó como referencia el diagrama del circuito presentado en la Figura 5, configurando el pin 10 de una tarjeta Arduino Uno, como salida digital. En estado alto, la salida digital suministró 5 voltios, mientras que en estado bajo, el suministro fue de 0 voltios. El voltaje en las terminales del condensador fue registrado por la entrada análoga A0 de la misma tarjeta. La tierra del circuito es la misma tierra o terminal GND de la tarjeta Arduino. Se utilizaron 6 condensadores de diferente capacitancia (ver Tabla 1).

\section{Primera serie de medidas}

\begin{tabular}{|c|c|c|c|c|}
\hline $\begin{array}{c}\text { CAPACITANCIA } \\
\text { NOMINAL }(\mu \mathrm{F})\end{array}$ & $\begin{array}{c}\text { CAPACITANCIA } \\
\text { MEDIDA CON LCR }(\mu \mathrm{F})\end{array}$ & $\begin{array}{c}\text { CAPACITANCIA } \\
\text { CALCULADA }(\mu \mathrm{F})\end{array}$ & RESISTENCIA $(\mathrm{K} \Omega)$ & $\begin{array}{c}\text { ERROR } \\
\text { PORCENTUAL }(\%)\end{array}$ \\
\hline 0.47 & 0,49 & 0,4948 & 104 & 0,97 \\
\hline 1 & 1,0574 & 1,0571 & 104 & $-0,03$ \\
\hline 4.7 & 4,44 & 4,382 & 104 & $-1,32$ \\
\hline 22 & 21,25 & 22,44 & 104 & 5,30 \\
\hline 47 & 42,42 & 42,58 & 12,2 & 0,38 \\
\hline 100 & 95,14 & 96,92 & 11 & 1,84 \\
\hline
\end{tabular}

Segunda serie de medidas

\begin{tabular}{|c|c|c|c|c|}
\hline $\begin{array}{c}\text { CAPACITANCIA } \\
\text { NOMINAL }(\mu \mathrm{F})\end{array}$ & $\begin{array}{c}\text { CAPACITANCIA } \\
\text { MEDIDA CON LCR }(\mu \mathrm{F})\end{array}$ & $\begin{array}{c}\text { CAPACITANCIA } \\
\text { CALCULADA }(\mu \mathrm{F})\end{array}$ & RESISTENCIA $(\mathrm{K} \Omega)$ & $\begin{array}{c}\text { ERROR } \\
\text { PORCENTUAL }(\%)\end{array}$ \\
\hline 0.47 & 0,49 & 0,488 & 90 & $-0,41$ \\
\hline 1 & 1,0574 & 1,045 & 90 & $-1,19$ \\
\hline 4.7 & 4,44 & 4,5024 & 11 & $-1,41$ \\
\hline 22 & 21,25 & 21,06 & 11 & $-0,90$ \\
\hline 47 & 42,42 & 47,079 & 11 & 9,90 \\
\hline 100 & 95,14 & 96,85 & 11 & 1,77 \\
\hline
\end{tabular}

Tabla 1. Comparación de valores obtenidos a partir de la ejecución del código con los valores registrados por medidores LCR

En la Tabla 1 se organizaron los resultados del experimento. El valor nominal es el que pone el fabricante en el cuerpo del condensador. La segunda columna corresponde a los valores proporcionados por el LCR, un instrumento especializado para determinar inductancia, capacitancia y resistencia. Se tomaron las mediciones de este instrumento como referencia para comparar con los datos de la tercera columna, que son los obtenidos a partir de la ejecución del programa, mostrado en la Figura 7. 
La Figura 8 presenta los valores obtenidos a partir de la ejecución del algoritmo en la tarjeta programable Arduino Uno, con una resistencia en serie de $10 \mathrm{~K} \Omega$ con un condesador de capacitancia nominal de $4,7 \mu \mathrm{F}$ y valor registrado en el medidor LCR de 4,44 $\mu \mathrm{F}$. Puede apreciarse que los valores de $\tau$ no son constantes pero oscilan alrededor de un promedio con baja dispersión

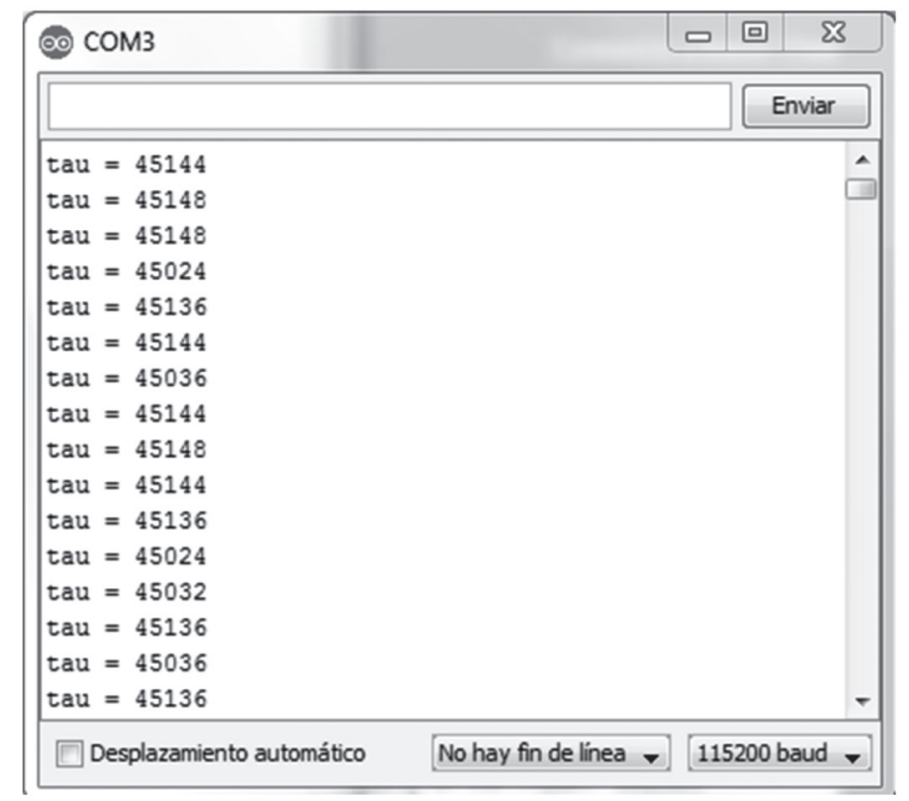

Figura 8. Registro de la constante de tiempo a partir del circuito de la Figura 5, ejecutando el algoritmo expuesto en la Figura 7

El valor visualizado en el monitor serial del entorno Arduino IDE es un entero que representa el tiempo en microsegundos. La constante de tiempo se calculó como el promedio aritmético de 100 valores sucesivos y posteriormente se determina $\mathrm{C}$ despejándo de la ecuación 3 y conocido el valor $\mathrm{R}$. El registro mostró que 10 de los 12 datos, presentaron un error porcentual menor del $\pm 2 \%$. El mayor desfase, ocurrió con el condensador cuya capacitancia nominal fue de $47 \mu \mathrm{F}$, donde se tuvo un error menor del $10 \%$. Con este condensador también se presentó un desfase mayor al $2 \%$ en la primera serie de medidas.

\section{CONCLUSIONES}

Con base en los resultados registrados en la Tabla 1, donde más del 80\% de las mediciones presentaron un error menor al $2 \%$ respecto a los valores registrados con el medidor LCR, puede afirmarse que el método de lectura de sensores capacitivos propuesto en el presente artículo tiene un grado de 
exactitud que le permite ser aplicado en sistemas mecatrónicos que generan acciones a partir de este tipo de transductores, aplicaciones que pueden ir desde la detección de nivel de líquido en tanques o reservorios, hasta la detección de posición en mecanismos móviles giratorios o lineales. En general, cualquier aplicación de detección de variables físicas con constantes de tiempo mayores como mínimo a 400 veces el ciclo de instrucción del dispositivo programable, se debe a que con este criterio se puede establecer teóricamente que el máximo tiempo de desfase está alrededor del $2 \%$.

No es recomendable utilizar el método aquí expuesto para implementar un instrumento de medición de capacitancias, pues el error porcentual está por encima del máximo considerado apropiado para un instrumento de medida que está determinado en 0.5\% (Creus, 2012; Wolf y Smith, 1992). Para lograr la exactitud y precisión consideradas aceptables para instrumentos de medición, sería necesario implementar el mismo circuito y el algoritmo con un dispositivo programable que tenga una frecuencia de reloj lo suficientemente alta para lograr los porcentajes normalizados. La tarjeta Arduino Uno trabaja con una frecuencia de reloj de $16 \mathrm{MHz}$, haciendo que cada ciclo de instrucción tenga una duración de $4 \mu \mathrm{S}$. Una estimación rápida indicaría que para lograr errores porcentuales menores a $\pm 1 \%$, tendría que ejecutarse el código en una máquina con un ciclo de instrucción de menos de $2 \mu \mathrm{S}$, es decir, con una frecuencia de reloj superior a $32 \mathrm{MHz}$.

El algoritmo propuesto y concretado en el código mostrado en la Figura 7 contiene pocas instrucciones y puede incorporarse fácilmente en una función de usuario, como un módulo que sería parte de un programa mayor, donde la detección de los cambios de capacitancia, como indicadores de la variación de alguna magnitud física, sería uno de los componentes. Un ejemplo puede ser la programación de un sistema de control de variables ambientales en un cultivo de invernadero. En este tipo de sistema, se controla la temperatura, la humedad relativa del aire, la humedad del suelo, la velocidad del caudal volumétrico de aire, la radiación de onda larga debido a la luz solar, entre otras. Para monitorizar todas estas variables se requiere de los sensores adecuados. Los sensores capacitivos detectan la humedad relativa, la humedad del terreno, el nivel de agua en los tanques de irrigación y en conjunto con los otros sensores, las electroválvulas, motobombas, calefactores, motores y demás actuadores, pueden componer un sistema automático de control para mantener las condiciones ambientales que garantizan la máxima productividad y calidad del cultivo. El programa que ejecute las tareas necesarias para hacer funcionar el sistema, puede estar compuesto de muchos módulos que incluyen aquellas fracciones de código dedicadas a estimar el valor de la variable en función de la detección de capacitancia de los sensores capacitivos disponibles. Otros módulos y rutinas, se encargarán de generar las acciones de control y corrección, manipulando los actuadores a través de la electrónica apropiada. 


\section{REFERENCIAS BIBLIOGRÁFICAS}

Baxter, L. K. (1997). Capacitive Sensors: Design and Applications (IEEE Press.). Nueva York: IEEE Press.

Chatzandroulis, S., Tsoukalas, D., y Neukomm, P. A. (2000). A miniature pressure system with a capacitive sensor and a passive telemetry link for use in implantable applications. Journal of Microelectromechanical Systems, 9(1), 18-23. doi:10.1109/84.825772

Chiang, C.-T., y Huang, Y.-C. (2006). A Semicylindrical Capacitive Sensor With Interface Circuit Used for Flow Rate Measurement. IEEE Sensors Journal, 6(6), 1564-1570. doi:10.1109/JSEN.2006.883847

Creus, A. (2012). Instrumentación Industrial. Marcombo.

Fernández, M., García, M., Alonso, G., Cano, J., y Solares, J. (1998). Técnicas para el mantenimiento y diagnóstico de máquinas eléctricas rotativas. Marcombo.

Huang, S. M., Stott, A. L., Green, R. G., y Beck, M. S. (1988). Electronic transducers for industrial measurement of low value capacitances. Journal of Physics E: Scientific Instruments, 21(3), 242. doi:10.1088/0022-3735/21/3/001

Jaworek, A., y Krupa, A. (2004). Gas/liquid ratio measurements by rf resonance capacitance sensor. Sensors and Actuators A: Physical, 113(2), 133-139. doi:10.1016/j.sna.2004.02.006

Kung, J. T., Lee, H.-S., y Howe, R. T. (1988). A digital readout technique for capacitive sensor applications. IEEE Journal of Solid-State Circuits, 23(4), 972-977. doi:10.1109/4.348

San Miguel, P. A. S. (2011). ELECTROTECNIA 2 BACH. Editorial Paraninfo.

Philipp, H. (1998, marzo 24). Time domain capacitive field detector. Recuperado a partir de http:// www.google.es/patents?id=QJAdAAAAEBAJ

Romani, A., Manaresi, N., Marzocchi, L., Medoro, G., Leonardi, A., Altomare, L., ... Guerrieri, R. (2004). Capacitive sensor array for localization of bioparticles in CMOS lab-on-a-chip. En Solid-State Circuits Conference, 2004. Digest of Technical Papers. ISSCC. 2004 IEEE International (pp. 224-225 Vol.1). doi:10.1109/ISSCC.2004.1332675

Tsugai, M. (1998, mayo 12). capacitive sensor interface circuit. Recuperado a partir de http://www. google.com.co/patents?id=ZFcjAAAAEBAJ

Wolf, S., y Smith, R. F. M. (1992). Guía para mediciones electrónicas y prácticas de laboratorio. Pearson Educación. 